\title{
Specificity of aza-peptide electrophile activity-based probes of caspases
}

\author{
KB Sexton ${ }^{1}$, D Kato ${ }^{1}$, AB Berger ${ }^{3}$, M Fonovic ${ }^{1,4}$, SHL Verhelst $^{1}$ and M Bogyo ${ }^{\star 1,1,23}$
}

Activity-Based Probes (ABPs) are small molecules that form stable covalent bonds with active enzymes thereby allowing detection and quantification of their activities in complex proteomes. A number of ABPs that target proteolytic enzymes have been designed based on well-characterized mechanism-based inhibitors. We describe here the evaluation of a novel series of ABPs based on the aza-aspartate inhibitory scaffold. Previous in vitro kinetic studies showed that this scaffold has a high degree of selectivity for the caspases, clan CD cysteine proteases activated during apoptotic cell death. Aza-aspartate ABPs containing either an epoxide or Michael acceptor reactive group were potent labels of executioner caspases in apoptotic cell extracts. However they were also effective labels of the clan CD protease legumain and showed unexpected crossreactivity with the clan CA protease cathepsin B. Interestingly, related aza peptides containing an acyloxymethyl ketone reactive group were relatively weak but highly selective labels of caspases. Thus azapeptide electrophiles are valuable new ABPs for both detection of a broad range of cysteine protease activities and for selective targeting of caspases. This study also highlights the importance of confirming the specificity of covalent protease inhibitors in crude proteomes using reagents such as the ABPs described here. Cell Death and Differentiation (2007) 14, 727-732. doi:10.1038/sj.cdd.4402074; published online 15 December 2006

Most proteolytic enzymes are regulated by a series of tightly controlled posttranslational modifications. Thus tools that can distinguish between active and inactive forms of proteases are of great value. Activity-based probes (ABPs) are small molecules carrying a reporter tag that often mimic a substrate and that specifically target and active site residues of an enzyme using a chemically reactive functional group. This reactive group is often referred to as a 'warhead' since it leads to permanent inactivation of the target by covalent modification of a primary catalytic nucleophile (for reviews, see Berger et al., ${ }^{1}$ Speers and Cravatt $^{2}$ and Verhelst and Bogyo ${ }^{3}$ ). ABPs that target a number of diverse protease families have been described, ${ }^{4}$ including a number of probes employing the acyloxymethyl ketone reactive warhead that label active caspases. ${ }^{5,6}$

The caspases are clan CD proteases whose concerted action is responsible for dismantling the cell during apoptotic cell death. ${ }^{7}$ Since the misregulation of apoptosis is central to the majority of human pathologies, ${ }^{8}$ significant effort has been devoted to the development of tools and reagents that facilitate the study of caspase-mediated cell death pathways. Recent work by Powers and co-workers has demonstrated the utility of aza-peptide electrophiles as potent and selective inhibitors of clan CD cysteine proteases. ${ }^{9-12}$ In particular, compounds containing an aza-asparate (aza-Asp) in the P1 position and either epoxide or Michael acceptor warheads were shown to be potent caspase inhibitors with very low crossreactivity towards other cysteine proteases. ${ }^{10,11}$
We recently developed a solid-phase synthesis methodology that permits facile introduction of multiple electrophilic warheads onto the aza-Asp peptide backbone. This method also allows rapid conversion of these inhibitors into ABPs by addition of a biotin tag. ${ }^{13}$ To assess the utility of this class of compounds as ABPs for the caspases, we synthesized a series of aza-Asp ABPs containing either epoxide, Michael acceptor or acyloxymethyl ketone (AOMK) warheads.

We report here that both aza-Asp epoxide and Michael acceptor ABPs potently label endogenous caspases in apoptotic cytosolic extracts. However, when these aza-Asp ABPs were incubated with proteomes containing high lysosomal protease activity, both probes efficiently labeled legumain and cathepsin B. Reactivity with the clan CA protease cathepsin $B$ was particularly unexpected as previous in vitro kinetic studies using purified enzymes identified these scaffolds as selective for inhibition of clan CD proteases. ${ }^{10,11}$ In addition, the novel class of aza-Asp AOMKs, while significantly less potent as ABPs, could be engineered to label multiple caspases by introduction of an optimized peptide binding sequence. These ABPs were more selective caspase labels, presumably as a result of their slow labeling kinetics. Thus we report here that aza-peptide electrophiles can be used both as broad-spectrum ABPs for multiple cysteine protease classes as well as specific ABPs for caspases.

\footnotetext{
${ }^{1}$ Department of Pathology, Stanford University School of Medicine, Stanford, CA, USA; ${ }^{2}$ Microbiology and Immunology, Stanford University School of Medicine, Stanford, CA, USA; ${ }^{3}$ Cancer Biology Graduate Program, Stanford University School of Medicine, Stanford, CA, USA and ${ }^{4}$ Department of Biochemistry and Molecular Biology, Jozef Stefan Institute, Ljubljana, Slovenia

${ }^{*}$ Corresponding author: M Bogyo, Department of Pathology, Stanford University School of Medicine, 300 Pasteur Drive, Stanford, CA 940305, USA.

Tel: + 650725 4132; Fax: + 650725 7424; E-mail: mbogyo@stanford.edu

Keywords: activity based probes; caspases; aza-peptides; cysteine protease; active site labels; protease inhibitors

Abbreviations: Z-VAD-FMK, benzyloxycarbonyl-VAD-fluoromethylketone; cbz, benzyloxycarbonyl; HRP, horseradish peroxidase; CHAPS, 3-[3-cholamidopropyl) dimethylammonio]-1-propanesulfonic acid

Received 25.9.06; revised 25.10.06; accepted 27.10.06; Edited by DR Green; published online 15.12.06
} 


\section{Results and Discussion}

Aza-Asp ABPs target endogenous caspases. We initially set out to assess the utility of the aza peptide electophiles as ABPs since this general class of compounds had been reported to show a high degree of potency and selectivity towards the caspase cysteine proteases involved in the process of programmed cell death. Using our solid phase method, we synthesized a series of ABPs containing the previously reported epoxide and Michael acceptor reactive groups. ${ }^{9,11}$ We also reasoned that since the peptide acyloxymethyl ketones (AOMK) were effective probes of caspases that an AOMK warhead could be used to make a potentially interesting new class of aza-peptide electrophiles. ${ }^{5}$ We therefore synthesized aza peptide ABPs containing either dimethyl or dichloro benzoic acid leaving groups to examine the effects of reactivity of the electrophile on target labeling. A biotin tag was linked through a hexanoic acid spacer to the primary peptide portion of probes that consisted of either the aza-Asp alone or the extended peptide sequence Glu-Val-azaAsp, shown previously to be optimal for multiple caspase family members (Figure 1). ${ }^{14}$

We first assessed the second-order rates of inhibition $\left(k_{2}\right)$ of the probes for recombinant caspase-3 (Table 1). Conversion of the previously published inhibitor, Cbz-EVaD-epoxide to the $A B P$ b-EVaD-epoxide resulted in less than a two-fold loss of potency. ${ }^{10}$ As expected, reduction of the peptide scaffold to the aza-Asp dramatically reduced the potency of the probe but a significant rate constant could still be measured. Interestingly, b-EVaD-epoxide was more potent than the previously reported peptide AOMK caspase probe b-EVD-AOMKMe while the aza-AOMKs did not produce measurable rate constants unless the extended EVaD sequence was used.

Based on the measured kinetic inhibition constants, bEVaD-epoxide would be predicted to be the most optimal ABP since it had the highest rate constant for the intended target. To test this hypothesis, we used our ABPs to label endogenous caspases in a cytosolic extract induced to undergo apoptosis by addition of cyctochrome $c$ and dATP (Figure 2a). This system enables direct activation of caspase9 and subsequently caspase -3 and -7 by promoting formation

Table 1 Second order rate constants for the Inhibition of caspase-3 by ABPs

\begin{tabular}{lc}
\hline Compound & $\boldsymbol{K}_{\mathbf{2}}\left(\mathbf{M}^{-\mathbf{1}} \mathbf{s}^{-\mathbf{1}}\right)$ \\
\hline b-aD-epoxide & $4241 \pm 345$ \\
b-EVaD-epoxide & $358233 \pm 14669$ \\
b-aD-Michael acceptor & $324 \pm 85$ \\
b-aD-AOMKCl & $<100$ \\
b-aD-AOMKMe & $\mathrm{NI}$ \\
b-EVaD-AOMKMe & $1832 \pm 137$ \\
b-EVD-AOMKMe & $20156 \pm 1507$
\end{tabular}

Values were obtained in caspase buffer using recombinant caspase-3 (see 'Materials and Methods'). $\mathrm{NI}=$ no inhibition

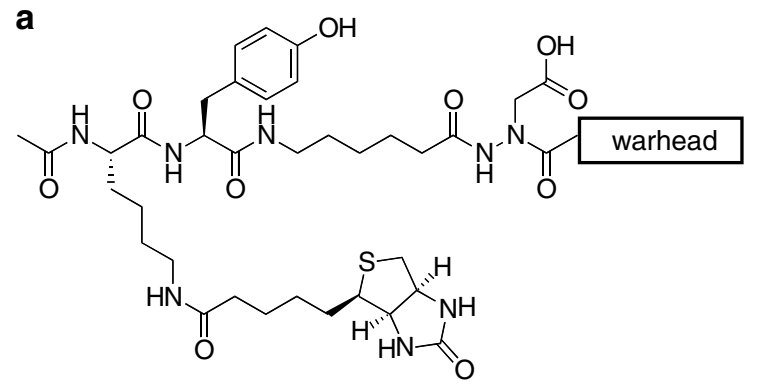

b

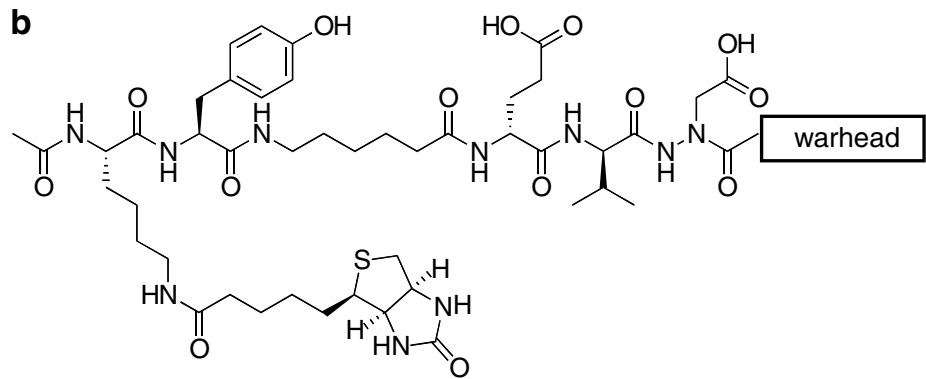
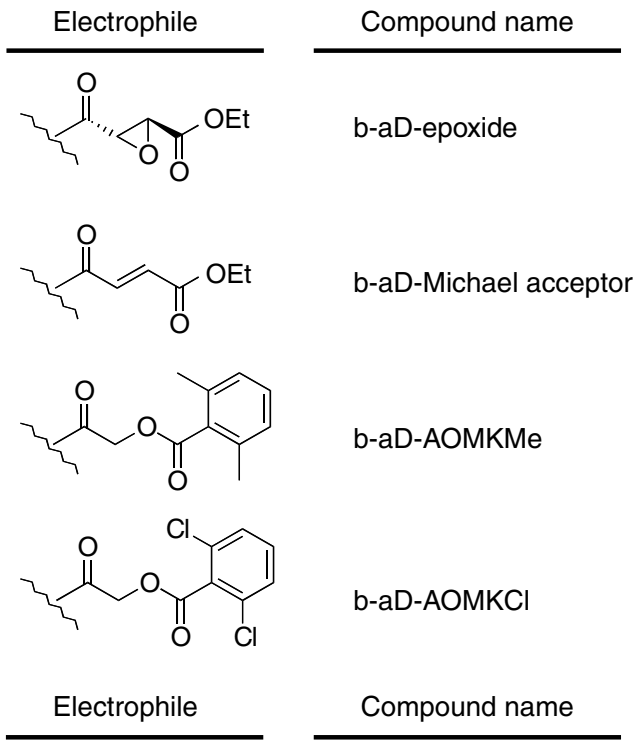

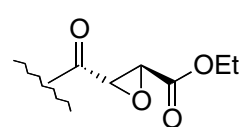

b-EVaD-epoxide<smiles>Cc1cccc(C)c1C(=O)OCC(=O)C(C)C</smiles>

b-EVaD-AOMKMe

Figure 1 Structure of aza peptide ABPs. (a) General scaffold for P1-only ABPs that contain an aza aspartic acid residue (aD). (b) Extended binding site ABPs that contain the Glu-Val-azaAsp peptide sequence (EVaD) 
a
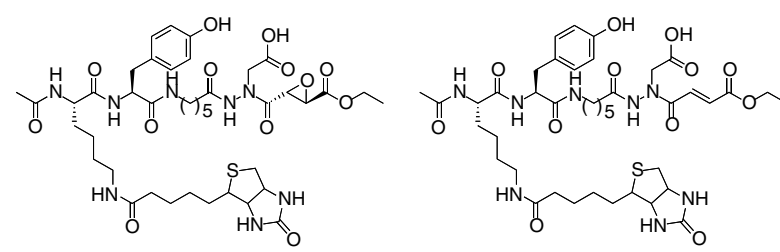

$$
10 \mu \mathrm{M} \quad 100 \mathrm{nM}
$$

cytc/dATP $-++\quad-++$

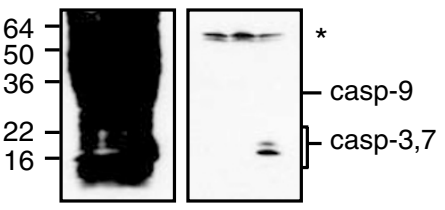

$\frac{\text { b-aD-Michael acceptor }}{10 \mu \mathrm{M} 100 \mathrm{nM}}$

$\begin{array}{ll}-++ & -+ \\ -+- & -+\end{array}$
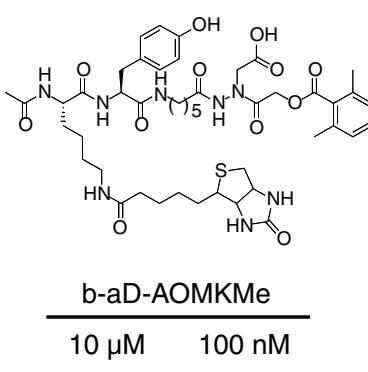

$-++\quad++$

$-+-+$

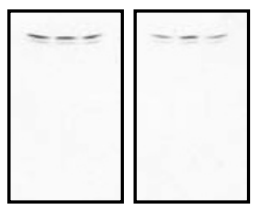

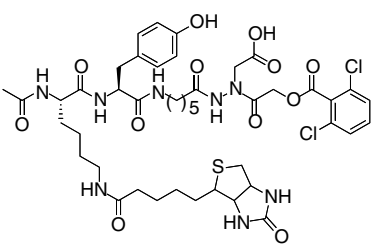

b-aD-AOMKCI

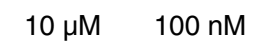

$-++\quad++$

- $-\quad-+$

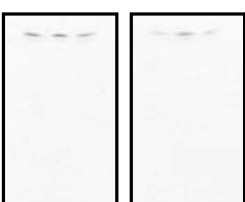

b
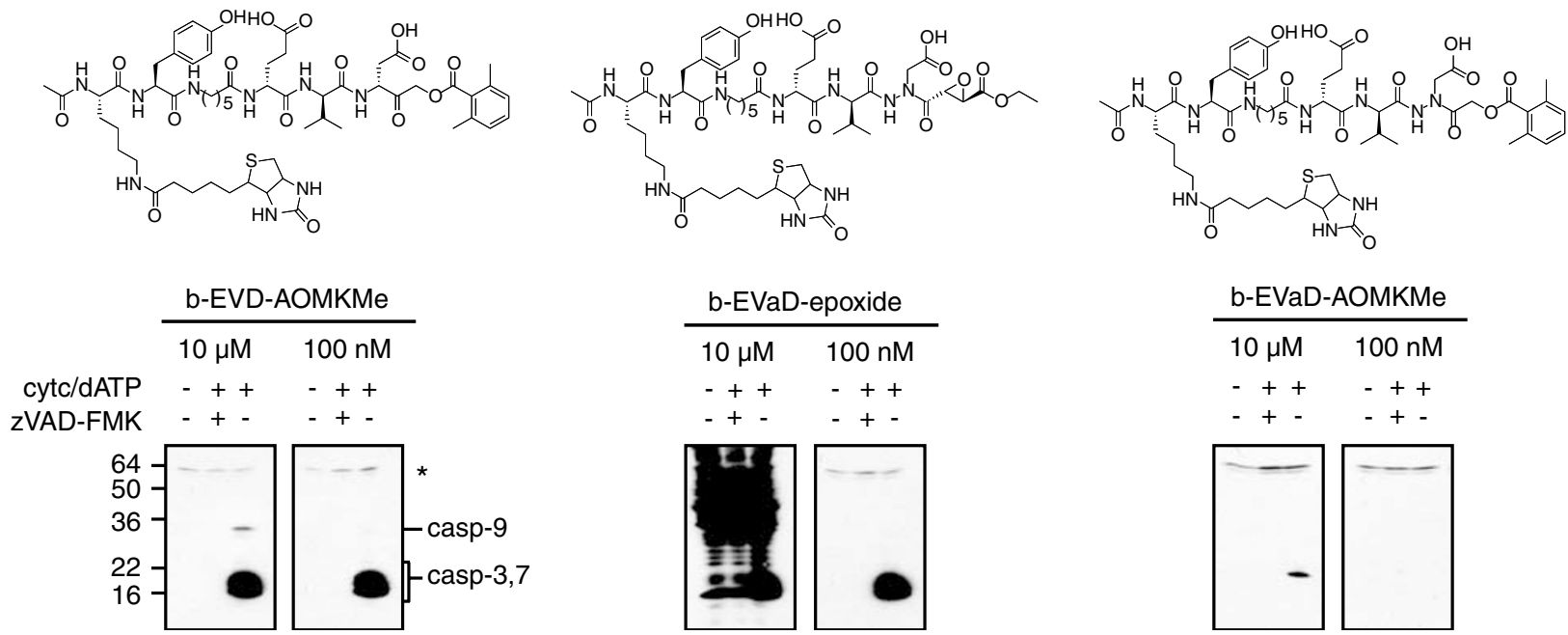

Figure 2 Detection of endogenous caspase activity. Caspase activation in cytosolic extracts of 293 cells was induced by the addition of cytochrome $c$ and dATP in the presence or absence of $50 \mu \mathrm{M}$ Z-VAD-FMK. Apoptotic extracts were labeled by addition of the P1-only aza peptidyl ABPs (a) or extended peptide ABPs (b) at low (100 nM) and high $(10 \mu \mathrm{M})$ concentrations. Active site labeling with b-EVD-AOMKMe is shown for comparison. Results from (a) and (b) were derived from the same experiment and western blot exposure, thereby allowing direct quantitative comparison. An endogenously biotinylated protein is indicated by $\left(^{*}\right)$

of the apoptosome. ${ }^{15}$ Addition of nanomolar concentrations of the b-aD-epoxide and b-aD-Michael acceptor ABPs resulted in selective labeling of executioner caspase- 3 and -7 . The identity of these targets was confirmed as mature processed forms of caspase- 3 and 7 using specific antibodies (data not shown). As expected, b-EVaD-epoxide was an effective label of mature caspase-3 and -7 and showed enhanced labeling of initiator caspase-9 (Figure 2b). This labeling by b-EVaDepoxide at 100 nanomolar concentration was similar to the previously characterized b-EVD-AOMKMe. ${ }^{5}$ However, the major difference between the peptide AOMK probe and its aza epoxide and Michael acceptor counterparts was the high degree of nonspecific reactivity observed for the aza probes when used at micromolar concentrations. Thus the aza epoxide and Michael acceptors, while potent inhibitors of caspases, are also highly reactive towards other protein targets thus potentially limiting their use as ABPs.

Not surprisingly, the aza-AOMK-based probes b-aDAOMKMe and b-aD-AOMKCl, which did not produce measur- able inhibition rate constants in our kinetic assays, showed no labeling of any caspases even at micromolar concentrations. However, the extended peptide probe b-EVaD-AOMKMe labeled caspase- 3 and -7, albeit less efficiently than its azaepoxide counterpart, when used at $10 \mu \mathrm{M}$. In direct contrast to b-EVaD-epoxide, this probe showed low background labeling even at high probe concentrations, possibly a function of its overall reduced reactivity.

Aza peptidyl ABPs crossreact with lysosomal proteases. Although the aza-Asp ABPs showed caspasespecific labeling in 293 apoptotic lysates when used at nanomolar concentrations at neutral $\mathrm{pH}$, it was not clear how these probes would behave at acidic $\mathrm{pH}$ in a proteome containing high levels of lysosomal protease activities. To determine probe specificity, we added ABPs to lysates from the macrophage-derived murine cell line RAW264.7, which contains high levels of both the clan CD protease legumain and the clan CA protease cathepsin B (Sexton and Bogyo, 
a

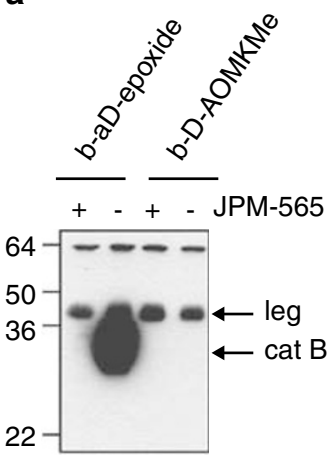

b

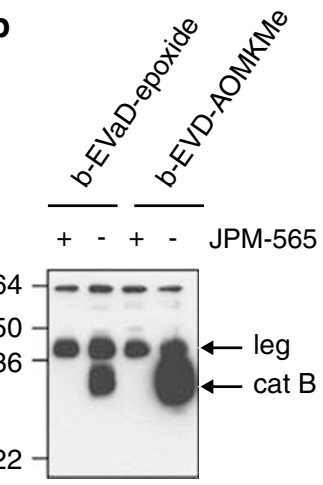

c
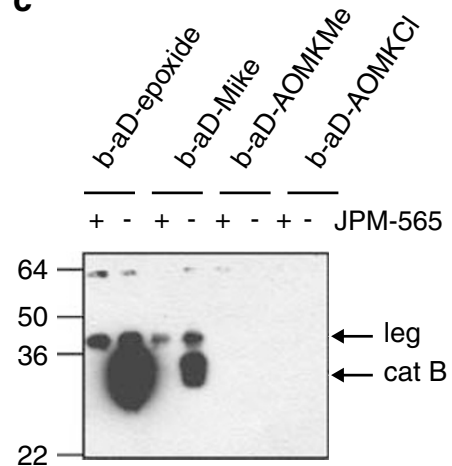

Figure 3 Detection of lysosomal protease activity using Aza epoxide probes. Detergent lysates from RAW264.7 cells prepared at pH 4.5 were incubated with $10 \mu \mathrm{M}$ probe in the presence or absence of $20 \mu \mathrm{M}$ JPM-565. (a) Direct labeling by b-D-AOMKMe and b-aD-epoxide. (b) Direct labeling by ABPs with extended peptide binding sites. Results in (a) and (b) are derived from the same experiment and western blot exposure. Note that extension of the peptide recognition region enhances cathepsin $B$ reactivity of the AOMK-based probe, while reducing cathepsin reactivity of the Aza epoxide probe. (c) Labeling of endogenous legumain and cathepsin activity using the P1-only aza peptidyl ABPs. Note the complete lack of cross reactivity of aza AOMK probes towards legumain or cathepsin B

unpublished observation). We have previously shown b-DAOMKMe to be an efficient and selective label of active legumain. ${ }^{5}$ We therefore compared reactivity between this $\mathrm{ABP}$ and our most reactive aza-Asp ABP, b-aD-epoxide (Figure $3 a$ ). Both probes efficiently labeled legumain activity as measured by the presence of a $37-\mathrm{kDa}$ protein that could not be blocked by the general papain family protease inhibitor JPM-565. Surprisingly, b-aD-epoxide showed extensive labeling of a $30-\mathrm{kDa}$ protease confirmed to be cathepsin $\mathrm{B}$ based on its size and sensitivity to cathepsin B specific inhibitors (data not shown). Furthermore, extension of the peptide binding scaffold of the two ABPs had opposite effects. b-EVD-AOMKMe showed enhanced labeling of cathepsin B compared to its P1-only counterpart, while bEVaD-epoxide showing reduced labeling (Figure $3 b$ ). This result clearly demonstrates that subsite specificity data cannot be extended a priori across classes of inhibitory scaffolds. In keeping with the overall reduced reactivity profile of the Aza-AOMK scaffold, b-aD-AOMK-Me did not show any crossreactivity with cathepsin or legumain targets.

Kinetic analysis of ABP reactivity with cathepsin $B$. The surprisingly efficient labeling of cathepsin $\mathrm{B}$ by $\mathrm{b}$-aD-epoxide, b-EVaD-epoxide and b-aD-Michael acceptor (Figure 3) prompted us to determine second-order inhibition values of these compounds against purified cathepsin B. In agreement with previous studies, we found that only the b-aD-epoxide had a detectable second-order inhibition constant (Table 2). We concluded that labeling of cathepsin $B$ reflected the cumulative effect of a slow labeling reaction between the ABP and the enzyme. This cumulative labeling effect is particularly relevant for production of probes for in vivo labeling studies where incubations are often carried out for several hours (Blum and Bogyo, unpublished obervation). To test the extent of this cumulative labeling effect on inhibition of cathepsin B, we set out to determine if these compounds could efficiently block activity of cathepsins in crude protein extracts following incubation for extended time periods.

To quantify the inhibitory potency of these probes towards endogenous lysosomal cathepsins, we used the well-
Table 2 Second order rate constants for the Inhibition of cathepsin B by ABPs

\begin{tabular}{lc}
\hline Compound & $\boldsymbol{K}_{\mathbf{2}}\left(\mathbf{M}^{-\mathbf{1}} \mathbf{s}^{-\mathbf{1}}\right)$ \\
\hline JPM-565 & $12287 \pm 930$ \\
d-aD-epoxide & $874 \pm 55$ \\
b-EVaD-epoxide & $\mathrm{NI}$ \\
b-aD-Michael acceptor & $\mathrm{NI}$ \\
b-aD-AOMKCl & $\mathrm{NI}$ \\
b-aD-AOMKMe & $\mathrm{NI}$ \\
b-EVaD-AOMKMe & $\mathrm{NI}$ \\
b-D-AOMKMe & $\mathrm{NI}$ \\
b-EVD-AOMKMe & $<500$ \\
\end{tabular}

Values were obtained in caspase buffer using recombinant caspase-3 (see 'Materials and Methods'). $\mathrm{NI}=$ no inhibition

characterized rat liver lysate proteome. ${ }^{16}$ Rat liver lysate was incubated with increasing concentrations of the ABPs for $60 \mathrm{~min}$ at $37^{\circ} \mathrm{C}$ prior to detection of residual active sites by labeling with the general papain family ABP ${ }^{125}$ I-DCG-04 (Figure $4 \mathrm{a}$ ). Crude $\mathrm{IC}_{50}$ values were calculated by direct quantification of the residual labeling by the radiolabeled probe (Figure 4b). The resulting inhibition data correlate extremely well with the labeling experiments (Figure 4c). All aza-Asp ABPs except the aza-AOMK-based probes showed unexpectedly efficient inhibition of cathepsin proteases. Likewise, truncation of the peptide binding sequence of the aza epoxides to the simple b-aD-epoxide significantly increased potency towards the cathepsins. Importantly, the b-aDepoxide probe was only sixfold less potent towards cathepsins $B$ and $Z$ and nearly as potent toward cathepsin $H$ as the optimal papain family inhibitor JPM-565. Thus, while overall kinetic inhibition rates may be low, when used for extended treatment times, the aza electrophiles are productive inhibitors of the papain family cathepsins. Furthermore, these data suggest that direct measurement of target binding in complex mixtures using a competition labeling method is likely to be a more informative measure of overall potency and selectivity of a covalent inhibitor or active site probe. Importantly, abundant enzymes such as cathepsin B have the potential to appear strongly labeled even when only a minor subset of enzyme 


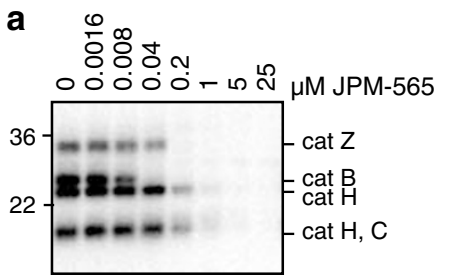

b

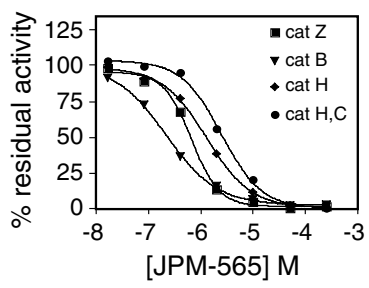

C

\begin{tabular}{|c|c|c|c|c|}
\hline \multirow[b]{2}{*}{ compound } & \multicolumn{4}{|c|}{$\mathrm{IC}_{50}(\mu \mathrm{M})$} \\
\hline & cat Z & cat B & cat $\mathrm{H}$ & cat $\mathrm{H}, \mathrm{C}$ \\
\hline JPM-565 & 0.066 & 0.022 & 1.3 & 2.5 \\
\hline cbz-EVaD-epoxide & 24.2 & 24.7 & $>25$ & $>100$ \\
\hline b-aD-epoxide & 0.373 & 0.136 & 2.0 & $>10$ \\
\hline b-EVaD-epoxide & $>100$ & 6.6 & $>100$ & $\mathrm{NI}$ \\
\hline b-aD-Michael & $>100$ & 61 & $>100$ & $>100$ \\
\hline b-aD-AOMKCI & $\mathrm{NI}$ & $\mathrm{NI}$ & $\mathrm{NI}$ & $\mathrm{NI}$ \\
\hline b-aD-AOMKMe & $\mathrm{NI}$ & $\mathrm{NI}$ & $\mathrm{NI}$ & $\mathrm{NI}$ \\
\hline b-EVaD-AOMKMe & $\mathrm{NI}$ & $\mathrm{NI}$ & $\mathrm{NI}$ & $\mathrm{NI}$ \\
\hline
\end{tabular}

Figure 4 Inhibition of endogenous cathepsins by aza epoxide probes and inhibitors. (a) Rat liver lysate was incubated with various concentrations of JPM-565 for $1 \mathrm{~h}$ at $37^{\circ} \mathrm{C}$. [ $\left.{ }^{125}\right] \mathrm{DCG} 04$ was then added and incubation continued for $45 \mathrm{~min}$ at $37^{\circ} \mathrm{C}$. Samples were resolved by SDS-PAGE and cathepsin activity was determined by autoradiography. (b) Quantification of $\mathrm{IC}_{50}$ values from SDS-PAGE data in (a). $\mathrm{IC}_{50}$ values were derived from a log plot of percentage competition versus probe concentration. Crude $\mathrm{IC}_{50}$ values were obtained from the linear portion of the plot. (c) Table of $\mathrm{IC}_{50}$ values (in $\mu \mathrm{M}$ ) for compounds obtained using the analysis shown in (a) and (b). NI indicates no measurable inhibition of labeling at up to $250 \mu \mathrm{M}$ compound

has reacted with the ABP. It is therefore imperative to quantify the extent of labeling as we have done here.

We have evaluated a series of ABPs that contain an azaAsp residue in the $\mathrm{P} 1$ position. We initially focused our efforts on the synthesis of this probe series because we believed, based on reported kinetic data, that it would provide highly potent and selective reagents for probing caspase biology. We were initially surprised to find that the first generation of Aza-Asp ABPs containing either epoxide or Michael acceptor warheads exhibited both high levels of background reactivity as well as crossreactivity towards the CA clan cathepsin protease cathepsin $B$, despite having very low second-order inhibition rates against the purified enzyme. Although these compounds are clearly useful as broad-spectrum ABPs, our results underscore the fact that neither ABPs nor covalent inhibitors can be selected based solely on kinetic characterization against purified enzymes. Nonethless, the potentially broad reactivity of the aza-peptide epoxides make them valuable reagents for proteomic analysis of multiple cysteine protease classes simultaneously using a single reagent. For these reasons, aza-peptide electrophiles represent a promising new class of reagents. Studies to validate these probes in proteomic and in vivo imaging applications are currently underway.

\section{Materials and Methods}

Reagents. The synthesis and characterization of all aza-Asp compounds has been previously described. ${ }^{13}$ b-D-AOMKMe and b-EVD-AOMKMe were synthesized as previously described. ${ }^{5}$ Z-VAD-FMK was purchased from MB pharmaceuticals.

Cell culture. 293 and RAW264.7 cells were cultivated in DME medium supplemented with $10 \%$ heat-inactivated bovine serum, $2 \mathrm{mM}$ L-glutamine, and penicillin/streptomycin. Cells were routinely passaged every 2-3 days.

Direct labeling of endogenous caspases. Cytosolic lysates from 293 cells were prepared as previously described. ${ }^{17}$ Apoptosis was induced by the addition of $10 \mu \mathrm{M}$ cytochrome $c$ and $1 \mathrm{mM} \mathrm{dATP}$ and incubation at $37^{\circ} \mathrm{C}$ for $30 \mathrm{~min}$ in the presence of $10 \mu \mathrm{M} \mathrm{z}$-VAD-FMK or DMSO control. ABPs were then added at the indicated concentrations and samples were incubated for an additional $60 \mathrm{~min}$ at $37^{\circ} \mathrm{C}$. Reactions were quenched by the addition of SDS-PAGE sample buffer. Samples were resolved by SDS-PAGE and transferred to a nitrocellulose membrane. Biotinylated proteins were visualized using streptavidin-HRP (500 ng/ ML, Sigma S-5512) and SuperSignal detection reagents (Pierce Chem. Co., Rockford, IL).

Direct labeling of endogenous cathepsin B and legumain. Confluent cultures of RAW 264.7 cells were mechanically removed from tissue culture flasks and washed three times with PBS. Cell pellets were incubated in $50 \mathrm{mM}$ citrate phosphate buffer (pH 4.5), $1 \%$ CHAPS, $0.5 \%$ Triton, $5 \mathrm{mM}$ DTT for $10 \mathrm{~min}$ on ice. Samples were centrifuged at $14000 \mathrm{~g}$ for $15 \mathrm{~min}$ at $4^{\circ} \mathrm{C}$ to remove cellular debris. The protein concentration of the supernatant was determined using the Bradford Assay (Pierce Chem. Co., Rockford, IL) and diluted to $20 \mathrm{mg} / \mathrm{mL}$ in lysis buffer. Samples were aliquoted and stored at $-80^{\circ} \mathrm{C}$ until the time of assay. Lysates $(49.5 \mu \mathrm{g})$ were prepared at a final volume of $49.5 \mu \mathrm{l}$ in reaction buffer $(50 \mathrm{mM}$ citrate phosphate buffer (pH 4.5), 0.1\% CHAPS, $5 \mathrm{mM} \mathrm{DTT}$ ) and incubated with $0.5 \mu \mathrm{l}$ of each compound diluted in DMSO to give the final concentration. Samples were resolved by SDS-PAGE and biotinylated proteins visualized as described above.

Determination of inhibition rates using the progress curve method. Bovine cathepsin B (Sigma) inhibition kinetics were determined as previously described. ${ }^{18}$ Recombinant human caspase-3 was expressed in $E$. coli and purified as described. ${ }^{19}$ Inhibition rates were determined as previously described. ${ }^{10}$

Determination of $\mathbf{I C}_{50}$ values. Detergent lysates were prepared from rat liver samples as previously described. ${ }^{16}$ Lysates were prepared in reaction buffer ( $50 \mu \mathrm{g}$ total protein in $50 \mu \mathrm{l}$ ) and incubated at $37^{\circ} \mathrm{C}$ for $1 \mathrm{~h}$ with $0.5 \mu \mathrm{l}$ of compound diluted in DMSO to give the desired final concentration. $\left[{ }^{125} \mathrm{~J}\right] \mathrm{DCG}-04\left(10^{6} \mathrm{cpm}\right)$ was then added and samples incubated for $45 \mathrm{~min}$ at $37^{\circ} \mathrm{C}$. Samples were resolved by SDS-PAGE and analyzed using a Typhoon 9410 imager (Scion Corp.). Densitometry was performed using $\mathrm{NIH}$ Image. $\mathrm{IC}_{50}$ values were determined from the linear portion of a plot of numerical values versus inhibitor concentration.

Acknowledgements. This work was supported by a Turman Fellowship at Stanford University (to M.B.), an NIH National Technology Center for Networks and Pathways Grant U54 RR020843-01 (to M.B.), NIH Grant R01 EB005011 (to M.B.), a Susan G Koman post-doctoral fellowship (to K.B.S.) and a National Human Genome Research Institute training Grant 5T32 HG00044 (to A.B.).

1. Berger $A B$, Vitorino $P M$, Bogyo M. Activity-based protein profiling: applications to biomarker discovery, in vivo imaging and drug discovery. Am J Pharmacogenom 2004; 4: 371-381.

2. Speers $A E$, Cravatt BF. Chemical strategies for activity-based proteomics. Chembiochem 2004; 5: 41-47.

3. Verhelst SH, Bogyo M. Chemical proteomics applied to target identification and drug discovery. Biotechniques 2005; 38: 175-177.

4. Evans MJ, Cravatt BF. Mechanism-based profiling of enzyme families. Chem Rev 2006; 106: 3279-3301.

5. Kato D, Boatright KM, Berger AB, Nazif T, Blum G, Ryan $C$ et al. Activity-based probes that target diverse cysteine protease families. Nat Chem Biol 2005; 1: 33-38. 
6. Thornberry NA, Peterson EP, Zhao JJ, Howard AD, Griffin PR, Chapman KT. Inactivation of interlukin-1 beta converting enzyme by peptide (acyloxy)methyl ketones. Biochemistry 1994; 33: 3934-3940.

7. Denault JB, Salvesen GS. Caspases: keys in the ignition of cell death. Chem Rev 2002 102: $4489-4500$

8. Reed JC. Mechanisms of apoptosis avoidance in cancer. Curr Opin Oncol 1999; 11 68-75.

9. Asgian JL, James KE, Li ZZ, Carter W, Barrett AJ, Mikolajczyk J et al. Aza-peptide epoxides: a new class of inhibitors selective for clan CD cysteine proteases. J Med Chem 2002; 45: 4958-4960.

10. James KE, Asgian JL, Li ZZ, Ekici OD, Rubin JR, Mikolajczyk J et al. Design, synthesis, and evaluation of aza-peptide epoxides as selective and potent inhibitors of caspases-1, - $3,-6$, and -8. J Med Chem 2004; 47: 1553-1574.

11. Ekici OD, Gotz MG, James KE, Li ZZ, Rukamp BJ, Asgian JL et al. Aza-peptide Michae acceptors: a new class of inhibitors specific for caspases and other clan CD cysteine proteases. J Med Chem 2004; 47: 1889-1892.

12. James KE, Gotz MG, Caffrey CR, Hansell E, Carter W, Barrett AJ et al. Aza-peptide epoxides: potent and selective inhibitors of Schistosoma mansoni and pig kidney legumains (asparaginyl endopeptidases). Biol Chem 2003; 384: 1613-1618.
13. Kato D, Verhelst SH, Sexton KB, Bogyo M. A general solid phase method for the preparation of diverse azapeptide probes directed against cysteine proteases. Org Lett 2005; 7: 5649-5652.

14. Thornberry NA, Rano TA, Peterson EP, Rasper DM, Timkey T, Garcia-Calvo M et al. A combinatorial approach defines specificities of members of the caspase family and granzyme B. Functional relationships established for key mediators of apoptosis. J Biol Chem 1997; 272: 17907-17911.

15. Liu X, Kim CN, Yang J, Jemmerson R, Wang X. Induction of apoptotic program in cell-free extracts: requirement for dATP and cytochrome c. Cell 1996; 86: 147-157.

16. Greenbaum D, Medzihradszky KF, Burlingame A, Bogyo M. Epoxide electrophiles as activity-dependent cysteine protease profiling and discovery tools. Chem Biol 2000; 7 569-581.

17. Stennicke HR, Jurgensmeier JM, Shin $H$, Deveraux $Q$, Wolf BB, Yang X et al. Pro-caspase3 is a major physiologic target of caspase-8. J Biol Chem 1998; 273: 27084-27090.

18. Blum G, Mullins SR, Keren K, Fonovic M, Jedeszko C, Rice MJ et al. Dynamic imaging of protease activity with fluorescently quenched activity-based probes. Nat Chem Biol 2005 1: 203-209.

19. Stennicke HR, Salvesen GS. Caspases: preparation and characterization. Methods 1999; 17: $313-319$ 